\section{Commentary: Duct stenting in neonates: Is it time to kiss goodbye our old friend, the Blalock-Taussig shunt?}

\author{
Majd Makhoul, MD, FAAP, FACC
}

Honjo and colleagues ${ }^{1}$ provide a meta-analysis and systematic review of the literature comparing 2 initial palliative approaches in infants with ductal-dependent pulmonary blood flow: stenting of arterial duct (DS) versus modified BlalockTaussig shunt (BTS).

After thorough review and strict inclusion criteria, they included 6 studies. The total number of patients was 514 in the BTS group and 246 in the DS group. Overall, the follow-up period was short- to midterm and 3 of the 6 studies had high bias. The studies reported somewhat different data points but the authors included those that were the most relevant.

DS patients had lower procedural complication rate and different types of complications compared with BTS patients. BTS patients had nonaccess-related bleeding as the most common complication $(\sim 10 \%)$, whereas DS patients had access-related vascular injury in $\sim 9 \%$ of cases. Procedural mortality rate (within 30 days of procedure) was not significantly different between the groups. Medium-term mortality rate was significantly lower in the DS group (relative risk, 0.66). DS patients had shorter intensive care unit and hospital length of stay. The risk of re-intervention in DS patients was significantly higher than in BTS patients $(47 \%$ vs $22 \%)$. Those included mainly balloon dilation and/or restenting of the duct. Quality of branch pulmonary arteries growth and need

\footnotetext{
From the Division of Pediatric Cardiology, Department of Pediatrics, University of Kentucky, Lexington, Ky.

Disclosures: The author reported no conflicts of interest.

The Journal policy requires editors and reviewers to disclose conflicts of interest and to decline handling or reviewing manuscripts for which they may have a conflict of interest. The editors and reviewers of this article have no conflicts of interest.

Received for publication July 6, 2020; revisions received July 6, 2020; accepted for publication July 7, 2020; available ahead of print July 14, 2020.

Address for reprints: Majd Makhoul, MD, FAAP, FACC, Department of Pediatrics, University of Kentucky, 138 Leader Ave, Lexington, KY 40506-9983 (E-mail: majd.makhoul@uky.edu).

J Thorac Cardiovasc Surg 2021;161:391-2

$0022-5223 / \$ 36.00$

Copyright (c) 2020 by The American Association for Thoracic Surgery

https://doi.org/10.1016/j.jtcvs.2020.07.025
}

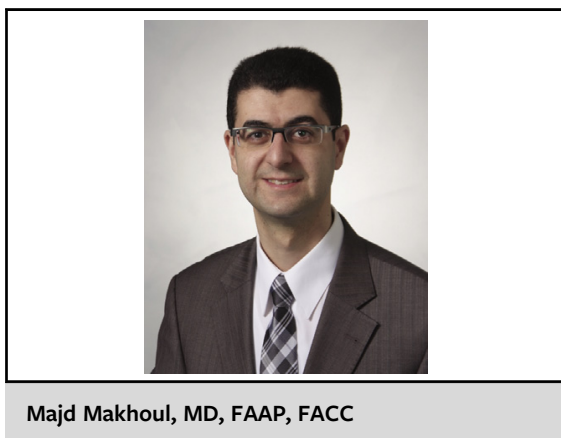

\author{
CENTRAL MESSAGE \\ Duct stenting is an effective, safe \\ alternative to Blalock-Taussig \\ shunt in infants with ductal- \\ dependent pulmonary blood \\ flow. RCTs are needed to define \\ the long-term superiority of \\ either approach.
}

for arterioplasty at time of next surgery was not different between the groups.

The article is a nice recap of the current evidence available on this topic. Although the quality of the studies included was overall low (observational studies with short periods of follow-up), they were very well selected and simply represent the best we have in the current era.

What I conclude from this review is that the DS is safe and effective option in experienced hands and produces quality of palliation at least as good as the surgical shunt. The high rate of reintervention in DS patients is to be expected due to in-stent stenosis as well as the growth of the patients and pulmonary arteries after the initial palliation. I can even say that reintervention should be planned ahead of time to achieve the biggest branch pulmonary arteries possible before the Glenn/hemi-Fontan procedure because this is a very important factor for future Fontan hemodynamics.

Another important variable is the morphology of the arterial duct because it varies in this patient cohort. Although the DS seems appealing and less invasive than BTS, defining the morphologic details of the duct and branch pulmonary arteries is very important step in patient selection. Some variations like discontinuous pulmonary arteries or significant duct tortuosity might favor the BTS because it provides more flexibility. 
An adequately powered randomized controlled trial is needed to tell which approach is superior. This will likely have to be expertise-based to minimize confounding factors like operator level of experience and participation enthusiasm.

DS has become a common, safe alternative to BTS in infants with ductal-dependent pulmonary blood flow, but the jury is still out as to whether or not there are long-term advantages for 1 approach versus the other.

\section{Reference}

1. Alsagheir A, Koziarz A, Makhdoum A, Contreras J, Alraddadi H, Abdalla T, et al. Duct stenting versus modified Blalock-Taussig shunt in neonates and infants with duct-dependent pulmonary blood flow: a systematic review and meta-analysis. J Thorac Cardiovasc Surg. 2021;161:379-90.e8.
See Article page 379.

\section{Commentary: Ductal stenting for ductal-dependent pulmonary blood flow: Time for an exclusive club to expand its membership?}

\author{
James M. Meza, MD, MSc, ${ }^{\text {a,b }}$ Neel Prabhu, BS, ${ }^{\text {a,c }}$ \\ Reid C. Chamberlain, MD, a,d,e \\ Joseph W. Turek, MD, PhD, ${ }^{\text {a,b,e }}$ and \\ Nicholas D. Andersen, MD ${ }^{\mathrm{a}, \mathrm{b}, \mathrm{e}}$
}

Infants born with ductal-dependent pulmonary blood flow (ddPBF) have been traditionally palliated initially with a systemic-to-pulmonary shunt, such as a modified Blalock-Taussig shunt (BTS). Stenting of the ductus arteriosus has been widely adopted, because it avoids the risks of a cardiopulmonary bypass and shunt thrombosis. In this systematic review and meta-analysis of 6 studies, Alsagheir and colleagues ${ }^{1}$ demonstrate that ductal stenting (DS) was associated with greater medium-term survival, lower number of complications, and intensive care unit and overall

From the ${ }^{a}$ Duke Congenital Heart Surgery Research and Training Laboratory, Durham, NC; ' Division of Thoracic and Cardiovascular Surgery, Department of Surgery, Duke University Medical Center, Durham, NC; ${ }^{\mathrm{c}}$ Duke University School of Medicine, Durham, NC; ${ }^{\mathrm{d}}$ Division of Pediatric Cardiology, Department of Pediatrics, Duke University Medical Center, Durham, NC; and 'Duke Children's Pediatric \& Congenital Heart Center, Durham, NC.

Disclosures: The authors reported no conflicts of interest.

The Journal policy requires editors and reviewers to disclose conflicts of interest and to decline handling or reviewing manuscripts for which they may have a conflict of interest. The editors and reviewers of this article have no conflicts of interest.

Received for publication July 20, 2020; revisions received July 20, 2020; accepted for publication July 22, 2020; available ahead of print July 24, 2020.

Address for reprints: James M. Meza, MD, MSc, 2301 Erwin Rd, Box 3443, Durham, NC 27705 (E-mail: james.meza@ duke.edu).

J Thorac Cardiovasc Surg 2021;161:392-3

$0022-5223 / \$ 36.00$

Copyright (c) 2020 by The American Association for Thoracic Surgery

https://doi.org/10.1016/j.jtcvs.2020.07.069

\section{Check for updates}

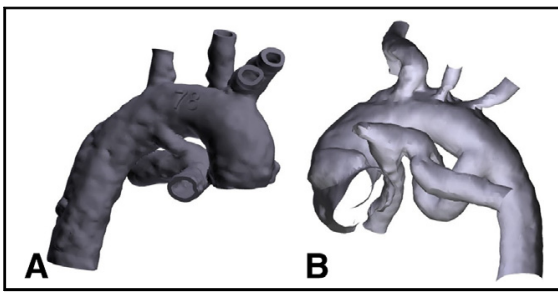

A, Good for stent: straight ductus, normal left pulmonary artery. B, Poor: tortuous ductus, left pulmonary artery from ductus.

\section{CENTRAL MESSAGE \\ In infants with ddPBF, DS may be preferable in suitable candidates. \\ Its use is especially attractive in SV disease and merits further study.}

length of stay. No difference was noted with 30-day survival, and DS was associated with a greater number of unplanned reinterventions. ${ }^{1}$

These pooled data begin to make the case that, in those who are appropriate candidates, DS is a preferable initial palliative strategy. Careful preprocedural planning, such as generating 3-dimensional angiographic models, is essential to maximize the likelihood of procedural success, so that these infants may reap the benefits of the lower morbidity and mortality profile of $\mathrm{DS}$. $^{2}$ While a useful additional approach, DS cannot completely replace BTS, because not all patients' ductal anatomy is amenable to stenting. Congenital heart surgeons should maintain their expertise in the technical complexities and postprocedural management of BTS placement.

It is important to note that all studies included in this review were retrospective cohort studies. The authors 